\title{
ENSAYO EXPERIMENTAL CON SUPERCONDENSADORES PARA SU UTILIZACIÓN COMO SISTEMA DE ALMACENAMIENTO DE ENERGÍA
}

José J. Quintana, Ignacio de la Nuez

ULPGC, Dpto. de Ingeniería Electrónica y Automática, josejuan.quintana@ulpgc.es, ignacio.nuez@ulpgc.es

Alejandro Ramos

Universidad de Las Palmas de G.C, Dpto. de Ingeniería de procesos, alejandro.ramos@ulpgc.es

Samuel Marrero

ULPGC, IUSIANI, samuelmarrero@siani.es

\begin{abstract}
Resumen
La creciente demanda de sistemas de almacenamiento de energía eléctrica ha provocado el desarrollo de muchos dispositivos diferentes. Ha habido un aumento en el uso de supercondensadores como sistemas de almacenamiento de energía, en particular los condensadores electroquímicos de doble capa (EDLC), que, debido a su densidad de energía y de potencia, compiten con las baterías y en muchas aplicaciones son complementarios con éstas. En este trabajo, en primer lugar se han diseñado unas pruebas experimentales y un prototipo, para obtener resultados estacionarios de carga, descarga y rendimientos de varios EDLC. Posteriormente, los EDLC se probaron con diferentes intensidades de carga y descarga para buscar la intensidad de prueba apropiada. Se llevaron a cabo una serie de pruebas con un EDLC de $10 F$, lo que permitió obtener los valores óptimos de la tensión de funcionamiento (mínimo y máximo), teniendo en cuenta la energía, la potencia y el rendimiento.
\end{abstract}

Palabras clave: Supercondensadores, EDLC, eficiencia energética, almacenamiento de energía eléctrica.

\section{INTRODUCCIÓN}

Las sociedades modernas utilizan nuevos dispositivos electrónicos para múltiples y diversas aplicaciones necesitando de sistemas aislados para almacenar la energía. Los dispositivos para el almacenamiento de energía eléctrica poseen dos características fundamentales para evaluar su eficacia y su eficiencia y son: la densidad de energía y la densidad de potencia. La densidad de energía define la capacidad de almacenar, teniendo en cuenta el volumen y el peso. La densidad de potencia define la energía por unidad de tiempo que se puede cargar o descargar.
Existen unas consideraciones generales adicionales que deben también considerarse, como son: el rendimiento del almacenamiento, la capacidad de almacenamiento (densidad de energía, número de ciclos y tiempo de suministro de energía a capacidad) y el rendimiento en la recuperación de la energía [1][2]. Un adecuado sistema de almacenamiento debe tener alto los dos parámetros indicados y normalmente, una situación de compromiso entre los valores de capacidad de almacenamiento y la densidad de potencia son necesarios para cada diseño.

Las baterías proporcionan una capacidad de almacenamiento de la energía alta pero una densidad de potencia no muy elevada, basándose su tecnología en reacciones químicas. El condensador tiene unas características complementarias a la batería y ofrecen una densidad de energía pequeña pero una elevada densidad de potencia [3]. El número de ciclos de operación en los condensadores en muy superior al alcanzado con las baterías y junto con la inexistencia de mantenimiento lo hacen recomendable para numerosas aplicaciones. Las habilidades para construir materiales con una elevada área interna y electrodos de baja resistencia han dado lugar a los supercondensadores, que acumulan mayor cantidad de energía, mediante la transferencia de carga entre un electrodo y un electrolito. Los supercondensadores, concretamente los condensadores electroquímicos de doble capa (EDLC), son dispositivos de reciente aparición, con una tecnología que proporcionan capacidades muy elevadas y ocupan unas especificaciones intermedias, ofreciendo altas capacidades de almacenamiento, inferiores a las baterías, junto con elevadas densidad de potencia, que se logra debido a la elevada superficie de los electrodos y al tamaño de los iones [4]. Todas las ventajas añadidas, (número de ciclos, inexistencia de mantenimiento, etc.), que dispone los condensadores electrolíticos son aplicables a los EDLC. 
El modelo eléctrico equivalente de estos dispositivos no es simple y numerosos trabajos se han desarrollados para identificar los parámetros más relevantes del comportamiento del mismo[5][6][7]. Sin embargo, las contribuciones de modelos para el almacenamiento energético son menores y mayoritariamente son sistemas eléctricos compuestos por resistencia y condensador únicamente [8]. Por otro lado, se han realizado, en el campo del almacenamiento energético de los EDLC, aportaciones para un adecuado diseño como sistema alternativo o híbrido de carga y descarga de energía[9][10].

Este trabajo muestra en el apartado dos el ensayo diseñado y las respuestas temporales de los EDLC que permiten obtener información relevante para el estudio energético. El tercer, cuarto y quinto apartado muestran los ensayos y los resultados realizados para la evaluación de los EDLC de 10F. En el último apartado se exponen las conclusiones.

\section{ENSAYO EXPERIMENTAL DE LOS EDLC}

Numerosos equipos de investigación han contribuido para conocer la dinámica de los EDLC diseñando prototipos experimentales, para la realización de ensayos, que permitan extraer comportamientos y conclusiones[11][12][13][14][15].

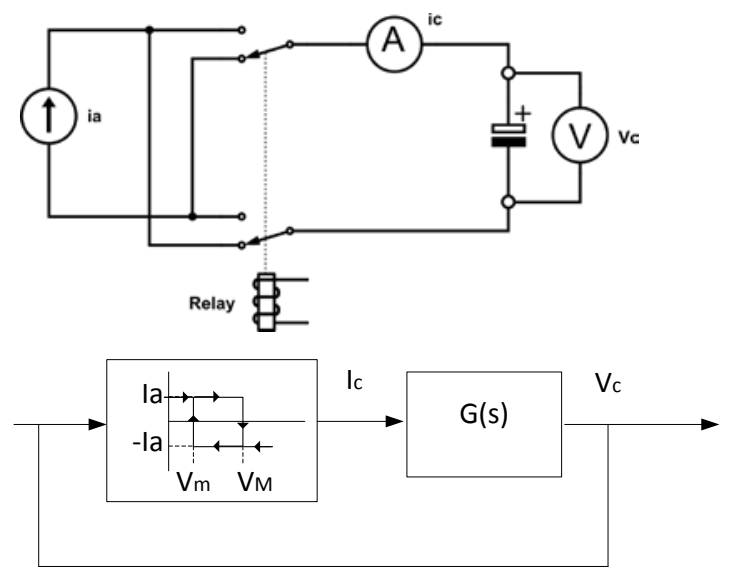

Figura 1: Esquema de potencia y control del circuito de pruebas.

En este trabajo se ha diseñado un prototipo para realizar ensayos con los EDLC utilizando un generador de corriente de intensidad máxima de 10A. Se ha utilizado una fuente programable de corriente, que en cada ensayo permanecerá constante, pudiéndose alternar entre valores positivos (carga del EDLC) y valores negativos (descarga del EDLC). El circuito eléctrico del prototipo de ensayos de los
EDLC se muestra en la figura 1. La adquisición de datos, (corriente y tensión), se obtiene con un período de 0,1s. El control realiza las funciones de carga, mientras el valor de la tensión en el condensador sea inferior a un valor de consigna máximo. Se invierte el signo de la corriente hasta que la tensión adquiere un valor mínimo de consigna, consiguiéndose que la tensión del condensador esté siempre entre los límites programados, $\left(V_{m} \leq V_{C} \leq V_{M}\right)$. Esta situación de carga y descarga permanecerá durante todo el ensayo, asegurándose que alcanza el régimen permanente y además, para asegurar la integridad del EDLC, se vigila que no se sobrepase la tensión máxima permitida por el fabricante. En la misma figura 1, se muestran los elementos más importantes del prototipo: Circuito de potencia y sistema de control.

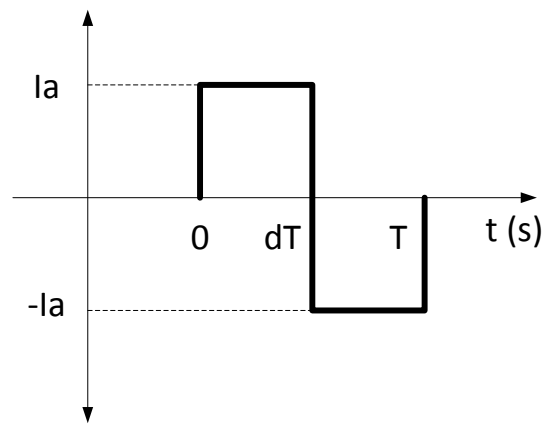

Figura 2: Evolución de la corriente en estado permanente.

La secuencia de las señales de entrada, pulsos de intensidad, permite su división en períodos, que constituyen el ciclo completo de la carga y descarga del EDLC. En esta situación y para un período, se tiene como señal de entrada un pulso como el representado en la figura 2. Este tipo de señal de entrada es fácil de realizar para los ensayos de laboratorio, ya que se consigue con un solo generador de intensidad. La señal definida en la figura 2 se puede descomponer en tres señales escalones simples, desplazadas en el tiempo,

$$
I_{C}(t)=I_{a} \cdot(u(t)-2 \cdot u(t-d T)+u(t-T))
$$

Siendo la función $u(t)$ la señal escalón unitario, que adquiere el valor unidad para cualquier valor positivo y cero para valores negativos. La amplitud de la intensidad que se aplica al EDLC es la definida por la fuente de valor $I_{a}$.

En cada ensayo, se aplica la señal de entrada indicada en (1) hasta que alcanza un estado permanente. El estado estacionario se consigue cuando el período de la intensidad, figura 2, se ha estabilizado, dando una respuesta de la tensión en el EDLC como la mostrada en la figura 3a y figura 3b. En la misma, figura 3c, se muestra la situación de régimen permanente que se utiliza para evaluar el rendimiento energético. En la 
misma figura, se definen dos intervalos: intervalo de carga de duración $[0, d T]$, donde $d \in[0,1]$, e intervalo de descarga de duración $[d T, T]$ segundos. Por lo tanto, para un EDLC de capacidad definida y una amplitud de la intensidad del ensayo dará un período diferente de régimen permanente. Como resultado de cada ensayo realizado, se obtienen el período de estabilización, es decir, el ciclo completo carga y descarga, y el intervalo de carga, que representa el tiempo que se ha estado aportando energía al EDLC. En la figura 3c, se pueden evaluar las dos áreas definidas para obtener la energía de entrada o de carga y la energía de salida o de descarga.
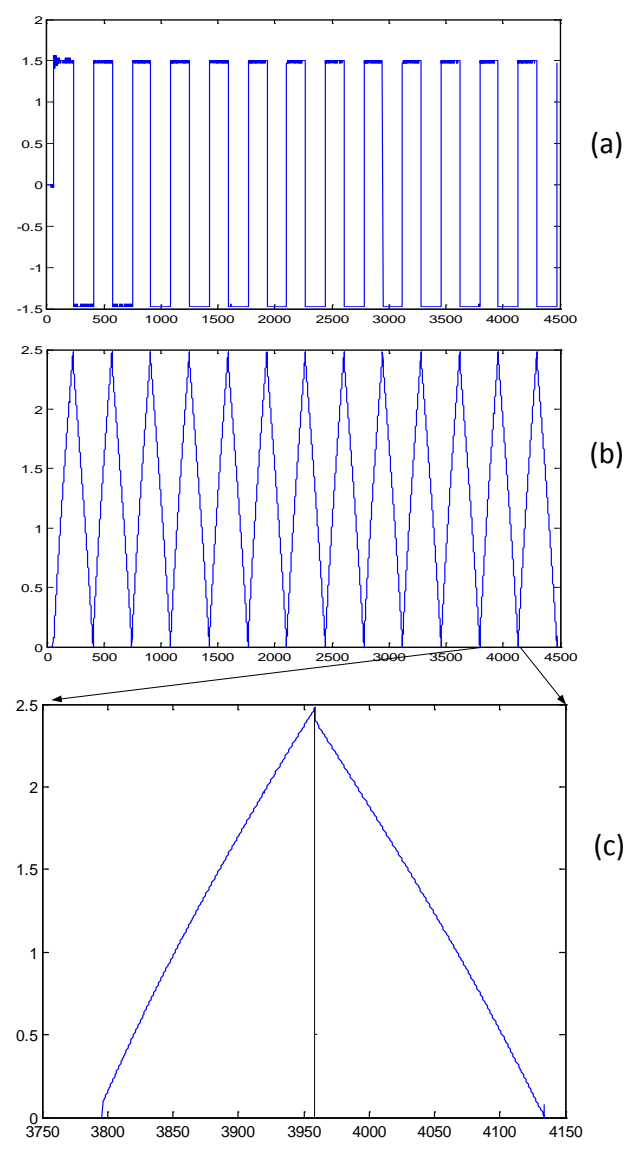

Figura 3: Detalle de la corriente y tensión en estado estacionario..

En el intervalo de carga, la energía de entrada se define como:

$$
E_{C}=\int_{0}^{d T} I_{C} \cdot V_{C} \cdot d t=I_{a} \int_{0}^{d T} V_{C} \cdot d t
$$

La intensidad en el ciclo de carga permanece constante, $I_{a}$, quedando la energía de entrada como el producto de la intensidad por el área definida por la tensión en el EDLC en el intervalo. De la misma forma se puede obtener la energía de descarga, que tiene el mismo valor de la intensidad pero de signo contrario, quedando:

$$
E_{D}=\int_{d T}^{T} I_{C} \cdot V_{C} \cdot d t=I_{a} \int_{d T}^{T} V_{C} \cdot d t
$$

El cálculo del rendimiento del sistema de almacenamiento se obtiene como cociente entre la energía de descarga dividido por la energía de carga.

$$
\eta=\frac{E_{D}}{E_{C}}=100 \frac{\int_{d T}^{T} V_{C} \cdot d t}{\int_{0}^{d T} V_{C} \cdot d t}
$$

El valor obtenido indica el máximo rendimiento en el EDLC en el proceso de carga y descarga sin que exista un intervalo de tiempo intermedio o autodescarga [16].
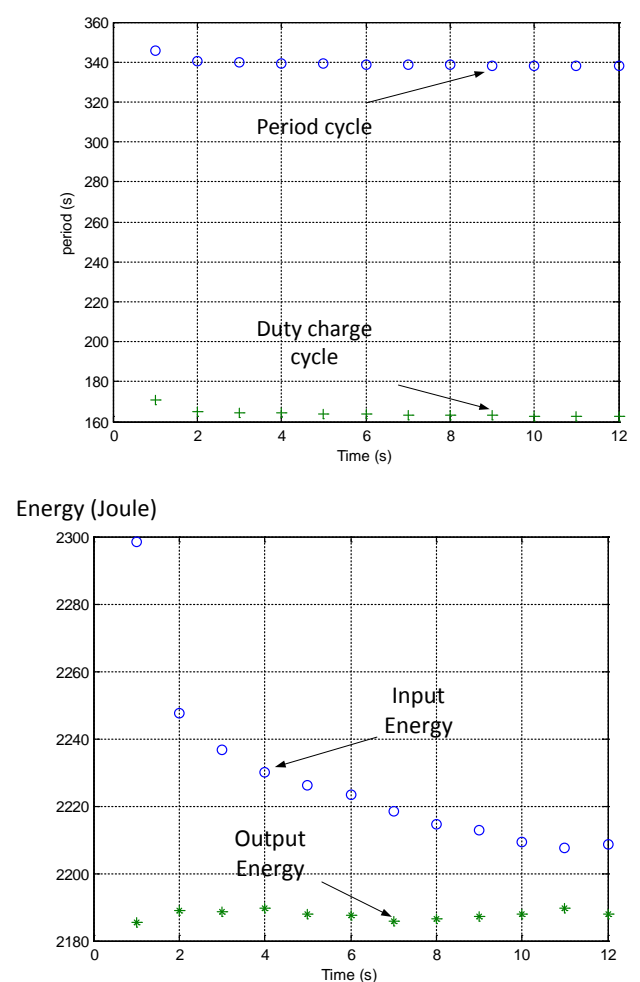

(a)

Figura 4: Evolución con el número de ciclo del periodo (a) y de la energía suministrada y recuperada (b).

En las figuras 4a y 4b se muestran para un ensayo genérico, la evolución del período y de las energías obtenidas. Cada ensayo comienza con el EDLC descargado, para asegurar la similitud de las respuestas con capacidades idénticas. Se observa, que el sistema se estabiliza al quinto período aproximadamente. En la figura $4 \mathrm{~b}$ se muestra la evolución de la energía de carga y descarga en el EDLC, y para los períodos posteriores al indicado. 


\section{3}

\section{EVALUACIÓN RENDIMIENTO EN LOS EDLC.}

La cantidad de energía máxima que se almacena en un EDLC está relacionada con la capacidad y la tensión máxima existente según la siguiente ecuación:

$$
E_{M}=\frac{1}{2} C \cdot V_{M}^{2}
$$

Siendo $E_{M}$ la máxima energía almacenada, C la capacidad y $\mathrm{V}_{\mathrm{M}}$ la tensión máxima en el EDLC. En el caso de los EDLC, la tensión varía, mientras se almacena o extrae la energía, pudiéndose extraer hasta que la tensión sea cercana a cero. Se define el parámetro $d_{S}$, como la relación entre la tensión mínima y la tensión máxima del EDLC,

$$
d_{S}=\frac{V_{m}}{V_{M}}
$$

Bajo estas condiciones se puede calcular la energía utilizable en un EDLC como:

$$
E_{u}=E_{M}\left(1-d_{S}^{2}\right)
$$

La mayoría de las aplicaciones con EDLC como dispositivo para almacenar energía, utilizan un valor, cociente de tensiones (6), comprendido entre 0,5 y 0,7 (50\% y $70 \%$ respectivamente) [1][17]. Con los valores de la relación de tensión indicados se consigue utilizar entre el $75 \%$ y el 51\% la energía almacenable en el EDLC.

En el apartado anterior se ha obtenido, mediante un ensayo experimental, una respuesta temporal que se puede utilizar para evaluar el dispositivo de almacenamiento. El EDLC ha almacenado energía en el primer tramo y devuelve la energía en el segundo tramo dentro del período. Las condiciones en este ensayo indican el máximo rendimiento posible, debido a que el EDLC ha almacenado energía e inmediatamente la descarga. En el ensayo indicado no se ha incluido ningún período de autodescarga que siempre influye disminuyendo el rendimiento. En el ensayo se programa los límites de la tensión mínima y de la tensión máxima y la amplitud de la intensidad. Se ha utilizado como tensión máxima en el EDLC la indicada por el fabricante de 2,5 voltios. El valor mínimo puede ser cualquier valor comprendido entre el rango de 0 hasta el valor máximo. Las diferentes posibilidades de la tensión mínima y la tensión máxima se han acotado para completar un número total de 15 ensayos, figura 5 . Los valores mínimos de la tensión varían desde el 0 hasta los 2 voltios con incremento de 0,5 voltios, dando un total de cinco valores diferentes. Para el valor máximo se ha realizado una variación desde los
0,5 voltios hasta los 2,5 voltios con incremento de 0,5 dando lugar a cinco valores diferentes. El valor mínimo de la tensión del ensayo y el valor de la diferencia de las tensiones del ensayo, que se define como la diferencia entre el valor máximo y el valor mínimo, representarán los dos ejes de coordenadas para su visualización. Con las variaciones indicadas de la tensión mínima y máxima, se tiene que la diferencia de las tensiones variará desde el valor de 0,5 hasta el valor de 2,5. Siempre se debe cumplir que la tensión mínima es inferior a la tensión máxima y el límite mínimo es de 0,5 voltios.

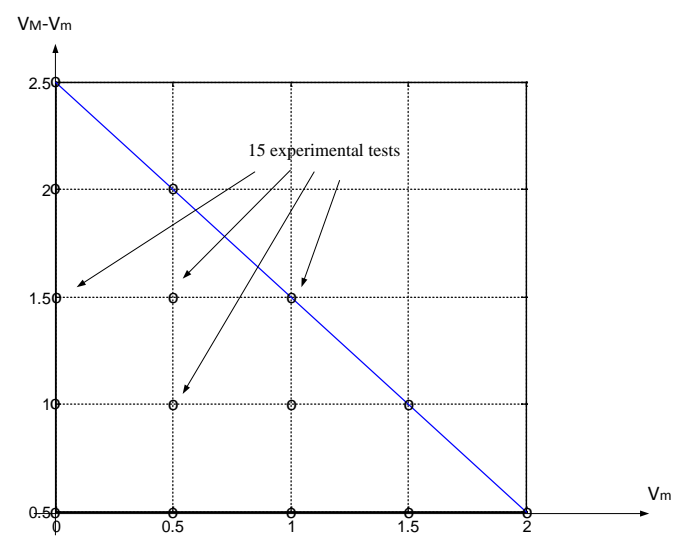

Figura 5: Valores máximos y mínimos de la tensión para las pruebas.

\section{SELECCIÓN DE INTENSIDAD DEL ENSAYO}

Las pruebas se han realizado con un EDLC de 10 Faradios. La primera parte consiste en localizar la intensidad apropiada del ensayo para la capacidad del EDLC. Dependiendo de la intensidad del ensayo se puede conseguir mejores rendimientos debido a que la frecuencia de la carga y descarga del EDLC varía y por lo tanto su rendimiento. El incremento de la intensidad genera una disminución del tiempo o del período de régimen permanente y no asegura una adecuada carga del EDLC. También está el proceso inverso, utilizando intensidades pequeñas se incrementa el tiempo necesario para realizar los dos intervalos de carga y descarga. Como primer conjunto de ensayos se han ensayado varios EDLC de igual capacidad a diferentes intensidades. Esta situación se ha realizado con una tensión mínima de cero voltios y una máxima de 2,5 voltios. En la tabla 1 , se muestran los resultados comenzando con la columna de la intensidad del ensayo $I_{\text {Test }}$. Las columnas segunda y tercera muestran el período en régimen permanente $T$ y el tiempo de carga en régimen permanente $t_{c}$. Las columnas cuarta y quinta representan la energía suministrada $E_{I}$ y extraída $E_{O}$ respectivamente. Por último, la columna sexta 
Tabla 1. Rendimiento en los EDLC de 10F variando la intensidad del ensayo

\begin{tabular}{|c|c|c|c|c|c|}
\hline $\begin{array}{c}\boldsymbol{I}_{\text {Test }} \\
(\mathbf{A})\end{array}$ & $\begin{array}{c}\boldsymbol{T} \\
(\mathbf{s})\end{array}$ & $\begin{array}{c}\boldsymbol{t}_{\boldsymbol{c}} \\
(\mathbf{s})\end{array}$ & $\begin{array}{c}\boldsymbol{E}_{\boldsymbol{I}} \\
(\mathbf{J})\end{array}$ & $\begin{array}{c}\boldsymbol{E}_{\boldsymbol{O}} \\
(\mathbf{J})\end{array}$ & $\begin{array}{c}\boldsymbol{\eta} \\
(\%)\end{array}$ \\
\hline 0,04 & 1315 & 655,6 & 33,79 & 31,62 & 93,6 \\
\hline 0,1 & 481,6 & 240,5 & 33,53 & 31,30 & 93,4 \\
\hline 0,25 & 189,9 & 94,9 & 32,61 & 30,04 & 92,1 \\
\hline 0,4 & 122,9 & 61,5 & 33,57 & 30,75 & 91,6 \\
\hline 0,5 & 98,2 & 49,1 & 33,29 & 30,29 & 91,0 \\
\hline 0,75 & 61,0 & 30,6 & 31,67 & 28,00 & 88,4 \\
\hline 1 & 45,8 & 23,0 & 31,45 & 27,14 & 86,3 \\
\hline 1,5 & 29,3 & 14,7 & 31,24 & 25,10 & 80,3 \\
\hline 4 & 10,2 & 5,2 & 31,13 & 20,27 & 65,1 \\
\hline
\end{tabular}

representa el rendimiento de este ensayo $\eta$. Como se observa en la tabla 1 , existe un máximo rendimiento entre los valores mínimos de 0,04 amperios hasta 0,5 amperios.

En la tabla 1 se muestra como varía el rendimiento en función de la intensidad del ensayo. Se reduce el rendimiento al mismo tiempo que el período también disminuye. Lo contrario se observa con intensidades pequeñas, el rendimiento es elevado al mismo tiempo que el período necesario aumenta considerablemente. Una situación intermedia se ha seleccionado de 0,4 A para la realización del estudio de los rendimientos energéticos de los EDLC de 10F.

\section{ENSAYO EXPERIMENTAL CON EL EDLC A LA INTENSIDAD DE 0,4A}

El ensayo experimental necesario para el estudio del rendimiento energético en los EDLC se basa en obtener los quince ensayos anteriormente explicados y su posterior evaluación. Los ensayos experimentales realizados con los EDLC de 10F se muestran en la tabla 2. La primera columna representa los diferentes valores que se han programado en el sistema de control para suministrar las tensiones mínimas y tensiones máximas en los ensayos. El conjunto de valores completan los quince ensayos con todas las posibilidades de la tensión mínima y máxima. En la segunda columna se muestra el período de cada ciclo en régimen permanente. En las tres columnas siguientes se muestran la energía de entrada, energía de salida y el cociente entre la energía de entrada y tiempo de la carga. En la última columna se muestra el rendimiento en cada uno de los ensayos.

Se observa que el rendimiento energético en este EDLC puede variar desde el 79,5\%, situación más desfavorable, hasta un 96,26 \%, situación con el
Tabla 2. Resultados de los ensayos para los EDLC de $10 \mathrm{~F}$ y $0,4 \mathrm{~A}$.

\begin{tabular}{|c|c|c|c|c|c|}
\hline $\begin{array}{c}\boldsymbol{V}_{\boldsymbol{m}}-\boldsymbol{V}_{\boldsymbol{M}} \\
(\mathbf{V})\end{array}$ & $\begin{array}{c}\mathbf{T} \\
(\mathbf{s})\end{array}$ & $\begin{array}{c}\boldsymbol{E}_{\boldsymbol{I}} \\
(\mathbf{J})\end{array}$ & $\begin{array}{c}\boldsymbol{E}_{\boldsymbol{o}} \\
(\mathbf{J})\end{array}$ & $\begin{array}{c}\boldsymbol{E}_{\boldsymbol{I}} / \boldsymbol{T} \\
(\mathbf{W})\end{array}$ & $\begin{array}{c}\boldsymbol{\eta} \\
(\%)\end{array}$ \\
\hline $0-0,5$ & 19,8 & 1,234 & 0,981 & 0,062 & 79,5 \\
\hline $0-1,0$ & 42,0 & 4,851 & 4,230 & 0,115 & 87,2 \\
\hline $0-1,5$ & 68,3 & 11,54 & 10,38 & 0,169 & 90,0 \\
\hline $0-2,0$ & 91,7 & 20,86 & 18,93 & 0,227 & 90,8 \\
\hline $0-2,5$ & 119,2 & 33,18 & 30,53 & 0,278 & 92,0 \\
\hline $0,5-1,0$ & 22,0 & 3,54 & 3,239 & 0,160 & 91,5 \\
\hline $0,5-1,5$ & 45,6 & 9,73 & 9,004 & 0,213 & 92,5 \\
\hline $0,5-2,0$ & 73,5 & 19,70 & 18,44 & 0,268 & 93,6 \\
\hline $0,5-2,5$ & 95,0 & 29,96 & 27,97 & 0,315 & 93,4 \\
\hline $1,0-1,5$ & 23,9 & 6,18 & 5,826 & 0,259 & 94,2 \\
\hline $1,0-2,0$ & 48,8 & 15,12 & 14,32 & 0,309 & 94,7 \\
\hline $1,0-2,5$ & 72,7 & 28,58 & 27,10 & 0,393 & 94,8 \\
\hline $1,5-2,0$ & 24,8 & 8,83 & 8,437 & 0,356 & 95,5 \\
\hline $1,5-2,5$ & 50,5 & 20,22 & 19,45 & 0,400 & 96,2 \\
\hline $2,0-2,5$ & 23,7 & 11,06 & 10,65 & 0,467 & 96,3 \\
\hline
\end{tabular}

mejor rendimiento. En la figura 6, se muestra el rendimiento energético en función de la tensión mínima y la diferencia entre la tensión máxima y mínima. Se observa que el rendimiento energético aumenta al aumentar la tensión mínima de carga, y que cuando ésta es cero, es decir, que el EDLC se descarga totalmente, el rendimiento baja drásticamente. Por tanto, se debe evitar que el EDLC se descargue totalmente en su operación normal. También se observa que el máximo rendimiento para una misma tensión mínima se da cuando la tensión máxima es $2,5 \mathrm{~V}$

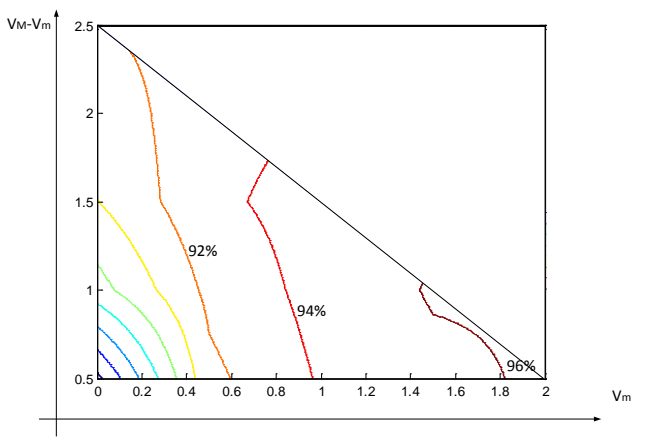

Figura 6: Relación entre el rendimiento y las tensiones máximas y mínimas del ciclo.

Sin embargo, se observa que en los ensayos con la energía de entrada máxima no coinciden con los ensayos de máximo rendimiento. Se observa que los ensayos que utilizan la máxima tensión son los que alcanzan la máxima energía de carga, llegando a 
conseguir los mejores rendimientos que varían desde el 92,01\% hasta el 96,26\%. También se ha obtenido el parámetro que define la energía por unidad de tiempo en estos ensayos como el cociente entre la energía de carga dividida por el intervalo de la carga, penúltima columna tabla 2. Extrayendo solamente los ensayos como límite superior el valor máximo de la tensión, se genera la tabla 3.

Tabla 3. Resultados de los ensayos para los EDLC de $10 \mathrm{~F}$ con los ensayos al valor máximo de 2,5 Volts.

\begin{tabular}{|c|c|c|c|c|c|c|}
\hline $\begin{array}{l}V_{m} \\
\text { (V) }\end{array}$ & $\begin{array}{c}T \\
(s)\end{array}$ & $\begin{array}{l}E_{I} \\
(J)\end{array}$ & $\begin{array}{c}E_{I} \\
(\%)\end{array}$ & $\begin{array}{c}E_{I} / T \\
\text { (W) }\end{array}$ & $\begin{array}{c}E_{I} / T \\
(\%)\end{array}$ & $\begin{array}{c}\text { Índice } \\
\text { (\%) }\end{array}$ \\
\hline 0 & 119,2 & 33,1 & 100 & 0,278 & 59,6 & 59,6 \\
\hline 0,5 & 95,0 & 30,0 & 90,3 & 0,315 & 67,6 & 61,0 \\
\hline 1 & 72,7 & 28,6 & 86,1 & 0,393 & 84,2 & 72,5 \\
\hline 1,5 & 50,5 & 20,2 & 60,9 & 0,400 & 85,8 & 52,3 \\
\hline 2 & 23,7 & 11,1 & 33,4 & 0,467 & 100 & 33,4 \\
\hline
\end{tabular}

En esta tabla 3 se han generado dos nuevas columnas. La columna cuarta, evalúa las pérdidas de almacenamiento de energía con respecto al ensayo de mayor capacidad (primera fila) y la columna sexta que, evalúa la disminución de la potencia con respecto al de mayor valor de potencia (última fila). Una situación de compromiso consiste en definir un índice como producto de ambas columnas indicadas, columna cuarta y columna sexta, para equilibrar las características de energía almacenada y energía almacenada por unidad de tiempo. La situación seleccionada corresponde al ensayo que utiliza como tensión mínima de un voltio proporcionando el máximo valor de los productos de ambos porcentajes.

\section{CONCLUSIONES}

Se ha diseñado un prototipo, fácilmente realizable, para el ensayo de los EDLC, que permite la obtención de las respuestas temporales necesarias para una evaluación del rendimiento energético. Los ensayos se han realizado para EDLC de $10 \mathrm{~F}$ de capacidad. Inicialmente, se ha localizado los valores de la intensidad de la carga y de la descarga que garantizan unos ensayos con rendimientos elevados y con tiempos de carga reducidos. Fijada la intensidad, se generan un total de 15 ensayos para mostrar las zonas de máximo rendimiento. Con la energía almacenada y la energía almacenada por unidad de tiempo se localizan las zonas de máximo rendimiento energético. Con estos ensayos se ha obtenido la información necesaria para la selección de la intensidad de la carga y de la descarga y las zonas de las tensiones recomendables en los EDLC. De la información obtenida, se observa que el rendimiento energético aumenta al aumentar la tensión mínima de carga, y que este rendimiento cae drásticamente si el EDLC se descarga totalmente.

Posteriores trabajos con EDLC deberán introducir diferentes capacidades, diferentes tipos o tecnologías de fabricación e introducir la autodescarga, para ir complementando la información necesaria de los EDLC para su uso como dispositivo de almacenamiento energético.

\section{English summary}

\section{TESTS ON EFFICIENCY OF SUPERCAPACITORS FOR USE AS ENERGY STORAGE SYSTEMS}

\begin{abstract}
The growing demand for storage systems of electric energy has resulted in the development of many different devices. There has been an increase in the use of supercapacitors as energy storage systems, in particular the electrochemical double-layer capacitors (EDLC), which, due to their quality of energy and power density, compete with batteries and in many applications they are complementary. Initially, experimental tests and a prototype were designed to gather stationary results for charge, discharge, and the EDLC's performance. Afterwards, the EDLCs were tested with different chargedischarge currents in order to achieve their suitable and appropriate current. A series of tests were carried out to an EDLC of $10 F$, obtaining the operating voltage's optimal values (minimum and maximum), taking into account the efficiency, the energy and the power.
\end{abstract}

Keywords: Supercapacitor, Efficiency energy, Electrical energy storing .

\section{Bibliografía}

[1] A. Burke, "Ultracapacitors: why, how, and where is the technology," J. Power Sources, vol. 91, pp. 37-50, 2001.

[2] P. Sharma and T. S. Bhatti, "A review on electrochemical double-layer capacitors,” Energy Convers. Manag., 2010.

[3] S. Westerlund and L. Ekstam, “Capacitor theory,” IEEE Trans. Dielectr. Electr. Insul., vol. 1, issue 5, pp. 826-839, 1994.

[4] B. E. Conway, Electrochemical supercapacitors: scientific fundamentals and 
technological applications. Plenum Press, 1999.

[5] L. Zubieta and R. Bonert, "Characterization of double-layer capacitors for power electronics applications,” Ind. Appl. IEEE Trans., vol. 36, no. 1, pp. 199-205, 2000.

[6] J. J. Quintana, A. Ramos, and I. Nuez, "Identification of the fractional impedance of ultracapacitors," in IFAC Proceedings Volumes (IFAC-PapersOnline), 2006, vol. 2, no. PART 1.

[7] J. J. Quintana, A. Ramos, and I. Nuez, "Modeling of an EDLC with fractional transfer functions using Mittag-Leffler equations,” Math. Probl. Eng., vol. 2013, 2013.

[8] A. Kuperman et al., "Supercapacitor sizing based on desired power and energy performance," IEEE Trans. Power Electron., vol. 29, no. 10, pp. 5399-5405, 2014.

[9] R. L. Spyker, "Classical equivalent circuit parameters for a double-layer capacitor," IEEE Trans. Aerosp. Electron. Syst., vol. 36, no. 3 PART 1, pp. 829-836, 2000.

[10] R. Chai and Y. Zhang, “A Practical Supercapacitor Model for Power Management in Wireless Sensor Nodes,” vol. 30, no. 12, pp. 6720-6730, 2015.

[11] C. H. Wu, Y. H. Hung, and C. W. Hong, "On-line supercapacitor dynamic models for energy conversion and management,” Energy Convers. Manag., 2012.

[12] N. Bertrand, J. Sabatier, O. Briat, and J. M. Vinassa, "Embedded fractional nonlinear supercapacitor model and its parametric estimation method," Ind. Electron. IEEE Trans., vol. 57, no. 12, pp. 3991-4000, 2010.

[13] A. Dzieliński, G. Sarwas, and D. Sierociuk, "Comparison and validation of integer and fractional order ultracapacitor models,” $A d v$. Differ. Equations, vol. 2011, no. 1, pp. 1-15, 2011.

[14] S. Buller, M. Thele, R. W. A. A. De Doncker, and E. Karden, "Impedance-based simulation models of supercapacitors and liion batteries for power electronic applications,” IEEE Trans. Ind. Appl., 2005.

[15] A. Burke and M. Miller, "Testing of electrochemical capacitors: Capacitance, resistance, energy density, and power capability,” in Electrochimica Acta, 2010.

[16] A. B. Cultura and Z. M. Salameh, "Modeling, Evaluation and Simulation of a Supercapacitor Module for Energy Storage Application,” 2015.

[17] A. Lewandowski, P. Jakobczyk, M. Galinski, and M. Biegun, "Self-discharge of electrochemical double layer capacitors," Phys. Chem. Chem. Phys., 2013.

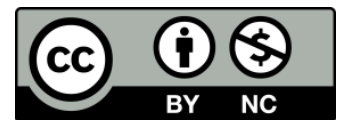

(C) 2018 by the authors. Submitted for possible open access publication under the terms and conditions of the Creative Commons Attribution CC-BY-NC 3.0 license (https://creativecommons.org/licenses/by-nc/3.0). 